\title{
Monitoring of clinical signs in goats with transmissible spongiform encephalopathies
}

\author{
Timm Konold ${ }^{1 *}$, Gemma E Bone¹, Laura J Phelan, Marion M Simmons, Lorenzo González², Sílvia Sisó \\ Wilfred Goldmann 3 , Saira Cawthraw ${ }^{4}$, Steve AC Hawkins ${ }^{1}$
}

\begin{abstract}
Background: As there is limited information about the clinical signs of BSE and scrapie in goats, studies were conducted to describe the clinical progression of scrapie and BSE in goats and to evaluate a short clinical protocol for its use in detecting scrapie-affected goats in two herds with previously confirmed scrapie cases. Clinical assessments were carried out in five goats intracerebrally infected with the BSE agent as well as five reported scrapie suspects and 346 goats subject to cull from the two herds, 24 of which were retained for further monitoring. The brain and selected lymphoid tissue were examined by postmortem tests for disease confirmation.

Results: The sensitivity and specificity of the short clinical protocol in detecting a scrapie case in the scrapieaffected herds was 3.9\% and 99.6\%, respectively, based on the presence of tremor, positive scratch test, extensive hair loss, ataxia and absent menace response. All BSE- and scrapie-affected goats displayed abnormalities in sensation (over-reactivity to external stimuli, startle responses, pruritus, absent menace response) and movement (ataxia, tremor, postural deficits) at an advanced clinical stage but the first detectable sign associated with scrapie or BSE could vary between animals. Signs of pruritus were not always present despite similar prion protein genotypes. Clinical signs of scrapie were also displayed by two scrapie cases that presented with detectable disease-associated prion protein only in lymphoid tissues.

Conclusions: BSE and scrapie may present as pruritic and non-pruritic forms in goats. Signs assessed for the clinical diagnosis of scrapie or BSE in goats should include postural and gait abnormalities, pruritus and visual impairment. However, many scrapie cases will be missed if detection is solely based on the display of clinical signs. $\mathrm{PrP}^{\mathrm{d}}$ accumulation in the brain appeared to be related to the severity of clinical disease but not to the display of individual neurological signs.
\end{abstract}

\section{Background}

Scrapie is a transmissible spongiform encephalopathy (TSE) of sheep and goats. The clinical presentation and progression of scrapie in sheep has been described in detail [1-3] whereas detailed descriptions of scrapie in goats are limited and often based on case reports [4-8] or experimental transmission of ovine or caprine scrapie to goats $[9,10]$. Similarly, the clinical signs of sheep experimentally infected with the bovine spongiform encephalopathy (BSE) agent have been documented in detail $[11,12]$ but the clinical picture of BSE in goats is less well defined: a short clinical course with ataxia and tremor was reported for intracerebrally inoculated

\footnotetext{
*Correspondence: t.konold@vla.defra.gsi.gov.uk

${ }^{1}$ Neuropathology, Veterinary Laboratories Agency Weybridge, New Haw, Addlestone, KT15 3NB, UK
}

animals whilst orally inoculated animals mainly presented with lethargy and weight loss resulting in recumbency over three weeks in the absence of ataxia. None of the goats showed signs of pruritus [13,14].

Monitoring of goats for TSE has recently become more relevant after naturally infected scrapie cases were retrospectively found to be infected with BSE [15] or presented with a BSE-like pathological phenotype [16] because BSE is currently the only TSE strain considered to be zoonotic [17]. Yet, compulsory TSE testing of slaughtered female goats over 18 months of age has been abolished since 26 June 2007 following the amendment (No 727/2007) of regulation (EC) No 999/2001 of the European Parliament laying down rules for the prevention, control and eradication of certain TSEs. As a consequence, the clinical examination has become more 
important to detect suspect cases of TSE in goats [18]. The objective of this paper is to describe the clinical signs and progression in goats affected by scrapie and BSE and to evaluate a short examination protocol to detect suspect cases in two herds of goats with a high incidence of scrapie.

\section{Methods}

All procedures were carried out in accordance with the Animal (Scientific Procedures) Act 1986, under Licence from the United Kingdom (UK) Government Home Office.

\section{Naturally infected cases (scrapie)}

Following the decision to cull two goat herds with confirmed scrapie cases, 150 goats from herd A and 196 goats from herd $\mathrm{B}$ were transported live to the Veterinary Laboratories Agency (VLA) Weybridge and clinically examined by a short protocol, as described below, prior to necropsy. Goats from herd A comprised 128 Saanen and 22 Alpine purebred or crossbred goats, 8-132 months old, 125 of which were female and 25 male; goats from herd B included 114 Anglo-Nubian, 49 Saanen, 22 Toggenburg and 11 Alpine purebred or crossbred goats, 11-115 months old, with one male and 195 female goats. Twenty-four of the 196 goats from herd B were retained for milk collection (17 goats) or monitoring of clinical progression (seven goats). All 346 goats of the herd cull were housed in groups of various size but never less than three.

Five scrapie suspect does from the same two herds (herd A: two Saanen, herd B: two Anglo-Nubian, one Saanen) that were transported to VLA Weybridge and kept for 1-27 days for clinical examination were also included.

\section{Experimentally infected cases (BSE)}

Five Saanen crossbred wethers from a herd without any previously known cases of scrapie were inoculated intracerebrally with $1 \mathrm{ml}$ of sterile bovine BSE brainstem as a $10 \%$ homogenate in physiological saline solution (BBP $12 / 92$ ) at 2 months of age under general anaesthesia. The inoculum consisted of five brainstems from BSEaffected cattle with a titre of $10^{3.3} \mathrm{lg}$ mouse i.c./i.p LD50 per $g$ tissue in RIII mice (for details on methods see [19]). Five days prior to inoculation, all goats were clinically examined to rule out clinical abnormalities. All goats were housed together; the last remaining goat was housed with sheep until cull.

\section{Clinical monitoring}

\section{Short clinical examination}

For the short examination protocol, animal handling was kept to a minimum: the assessment of the menace response, scratch test and hair loss and body condition score (BCS) and other parameters (behaviour, locomotion) were assessed in the pen (see additional file 1: Short exam protocol). This protocol was used for all 346 goats from the herd cull, within five days of arrival at VLA although the median times from examination to necropsy were 8 days in herd A and 26 days in herd B. As some of the goats from herd $B$ were lactating, behaviour during milking was also recorded by husbandry staff.

\section{Long clinical examination}

The long examination protocol comprised a physical and neurological assessment, which included assessment of behaviour, cranial nerve function, proprioception, locomotion in a corridor, muscle movements and signs of pruritus, such as hair loss and scratch response testing, similar to the clinical examination protocol used for sheep [12] (see additional file 2: Long exam protocol). This protocol was used for the five scrapie field suspects (single examination in four, two examinations in one) and for the five BSE-infected goats, which were examined quarterly from 15 months post inoculation (mpi) or more frequent depending on clinical progression.

Goats that were part of the herd cull and were later retained for milk collection or monitoring of clinical progression were re-examined subsequently using the short protocol only (eight goats, re-examined once) or the long protocol (16 goats, examined up to five times at 2-4 weekly intervals).

For the purpose of this report, a positive scratch test was defined as a stereotypical response to scratching of the dorsum, characterised by head movements with ("nibble reflex") or without lip movements, which was elicited repeatedly and consistently [12]. Any such response that was inconsistently exhibited as well as crouching in response to scratching was considered an inconclusive scratch test result. As the scratch test required the animal to be calm, it was not performed on those animals that were too nervous or restless (= unable to test). Skin abnormalities included abrasions, lichenification, papules and scabs, which have been associated with scrapie in sheep $[2,20]$. The menace response was "absent" if it was not elicited uni- or bilaterally despite apparently normal ocular structures and "exaggerated" if the animal responded repeatedly with a head flinch or toss. A hunched appearance, crouching or standing with the legs wide-based was considered an abnormal posture. Spontaneous startle was any flinch or startle that was elicited without obvious stimulus or occurred to stimuli that were considered to be familiar to goats (e.g. approaching an animal); it was a repeated response if it occurred more than once.

The BCS was evaluated on a scale from 0 to 5 as for sheep [21]; BSE-inoculated goats were weighed monthly from 2 mpi and prior to cull. 


\section{Passive behavioural observations recorded on camera}

In addition to the clinical examinations, the behaviour and activity of all BSE-affected goats, two reported scrapie suspects from herd A and 17 goats from herd B in their pens were recorded on video (digital video camera recorder DCR-TRV950E, Sony, Weybridge, UK, or wireless network camera 207W, Axis Communications, Lund, Sweden) for a minimum of 40 minutes.

Pruritic behaviour recorded via cameras was grouped into rubbing (rubbing parts of body on fixed objects), nibbling (nibbling of body parts) and scratching (scratching of body parts with limbs or horns). All pruritic behaviour per observation period was subsequently added up and divided by the observation time to obtain a value (pruritus score $=$ pruritic session per minute) for comparison between days or animals. The pruritic score was calculated for each BSE-infected goat from the last camera recordings prior to cull whereas all camera recordings from arrival at VLA to cull were taken into consideration for the goats from the herd cull and one scrapie suspect from herd A, which was kept for 27 days.

Based on the clinical presentation animals were grouped into three categories: no obvious signs, inconclusive (signs may be caused by a TSE) and definite signs. Definite signs of a TSE were present if animals displayed pruritic behaviour (positive scratch test or extensive hair loss with or without skin lesions due to rubbing), which was suggestive of the scratching syndrome, whereas the presence of ataxia and tremor was suggestive of the nervous syndrome [9]. The presence of single signs like tremor (mainly involving the whole body or the legs), ataxia, pruritic behaviour [pruritus score $\geq 0.5$ (= one or more pruritic activities every 2 minutes) in the absence of a positive scratch test or extensive hair loss], abnormal behaviour during milking, such as nervousness with trembling, kicking during milking or reluctance to enter the milk stand, and an absent menace response despite apparent normal vision, were interpreted as inconclusive signs with respect to TSE.

For the evaluation of the short examination protocol to detect suspect scrapie cases, only those signs that could be observed during the assessment were taken into consideration to assess the clinical TSE status; for example, an animal that was difficult to milk but unremarkable during the short examination was considered to be free from scrapie.

\section{Disease confirmation}

Diagnosis of the five scrapie suspects was based on the histopathological and immunohistochemical examination (IHC) of the formalin-fixed brainstem at the level of the obex for presence of vacuolation and diseaseassociated $\operatorname{PrP}, \operatorname{PrP}^{\mathrm{d}}$, using antibody R145 (VLA
Weybridge, Addlestone, UK [22]). Palatine tonsil and recto-anal mucosa-associated lymphoid tissue (RAMALT) were also examined by IHC using the same antibody. In addition, samples of the medulla caudal to the obex were examined for the presence of proteinaseresistant PrP, PrP ${ }^{\text {res }}$, by Western immunoblot (VLA Hybrid technique [23]) and ELISA (TeSeE ELISA, BioRad Laboratories Ltd, Hemel Hempstead, UK).

Samples of medulla at the obex, medial retropharyngeal lymph node and RAMALT from 111 goats from herd A were examined by IHC with PrP antibody Bar224 (CEA, Paris, France [16]). The same IHC protocol was applied to a much wider range of tissue samples of 39 goats from herd A and all 196 goats from herd B; in addition to the three tissue samples above mentioned, sections of diencephalon, thoracic spinal cord, distal ileum, palatine tonsil, spleen, nictitating membrane, prescapular, distal jejunal and pre-femoral lymph nodes, were also examined. In 74 goats (two from herd $\mathrm{A}$ and 72 from herd B), a more detailed pathological examination of the brain was performed in ten specific brain areas (cerebral cortex, corpus striatum, septal area, thalamus, hypothalamus, tegmentum, substantia nigra, cerebellum, ventral medulla and obex), where the accumulation of $\mathrm{PrP}^{\mathrm{d}}$ was subjectively scored from 0 (nil) to 3 (severe). Particular attention was given to the thalamus because it regularly presented with profound neuropathological changes in experimental scrapie of goats, which were associated with pruritus, and to the cerebellum because progressive incoordination of voluntary movements and tremor observed in experimental caprine scrapie could suggest pathological changes in these structures [10].

Confirmation of BSE was based on histopathological examination and IHC of the brain at the level of the obex; other brain tissues were frozen and thus not available for histopathological examination. Discrimination of BSE and scrapie was based on immunolabelling pattern by IHC using antibodies R145 and P4 (R-Biopharm AG, Darmstadt, Germany [16]). Tissues of the lymphoreticular system (LRS) examined by IHC with antibody R145 were the medial retropharyngeal and mesenteric lymph nodes, RAMALT and palatine tonsil.

\section{Genotyping}

Blood samples from the goats were collected to determine the full open reading frame of the caprine prion protein gene (PRNP) from codon 1 to codon 256. Genotyping for goats from the herd cull was conducted as described previously [24]. A slightly modified protocol was used for genotyping of the scrapie suspects and the BSE-inoculated goats: Polymerase chain reaction (PCR) was conducted on a GeneAmp ${ }^{\circledR}$ PCR System 9700 (Applied Biosystems Inc., Foster City, USA) in a $100 \mu \mathrm{l}$ 
final reaction volume, containing $1 \times$ GeneAmp $^{\circ}$ PCR Buffer (with $1.5 \mathrm{mM} \mathrm{MgCl}$ ) (Applied Biosystems Inc.), $200 \mu \mathrm{M}$ dNTPs, $0.03 \mathrm{U} / \mu \mathrm{l}$ AmpliTaq Gold ${ }^{\circledR}$ DNA Polymerase (Applied Biosystems Inc.), 500 nM PCR primers and $8 \mu \mathrm{l}$ of extracted genomic DNA sample. Primers for the PCR were 5' TGGAATGTGAAGAACATTTAT GACCTAGAAT 3' (Position 391-421 [GenBank EU032304/EU032305]) and 5' CAAGAGAGAAGCAAGAAATGAGACA 3' (position 1387-1411 [GenBank EU032304/EU032305]). PCR conditions were one cycle of 10 minutes at $95^{\circ} \mathrm{C}, 40$ cycles of 30 seconds at $95^{\circ} \mathrm{C}$, 30 seconds at $62^{\circ} \mathrm{C}$ and 1 minute at $72^{\circ} \mathrm{C}$ and a final 10 minute elongation at $72^{\circ} \mathrm{C}$. The reactions were purified using HTS PCR 96 Well purification plate system (Millipore, Billerica, USA) and then sequenced using the sequencing primers 5' AACCAACATGAAGCATGTGG 3' (position 859-878 [GenBank EU032304/EU032305]) and 5' GGTGAAGTTCTCCCCCTTGGT 3' (position 1118-1138 [GenBank EU032304/EU032305]) on the ABI 3730xl Genetic Analyzer with the Big Dye Terminator v3.1 Cycle Sequencing Kit (Applied Biosystems Inc.) as per manufacturer's instructions.

\section{Statistical analysis}

Individual signs assessed at the short clinical assessment were compared between TSE-positive (CNS and/or LRS positive) and TSE-negative goats by Fisher's exact test.

\section{Results}

\section{Naturally infected cases (scrapie)}

\section{Evaluation of the short protocol to detect scrapie cases}

The pathological findings and its association with PRNP genotype and age in herd $B$ have been described in detail elsewhere: there was no effect of age on disease prevalence (as determined by postmortem tests) in isoleucine homozygous goats $\left(\mathrm{II}_{142}\right)$, which was contrary to methionine carriers $\left(\mathrm{IM}_{142}\right.$ or $\left.\mathrm{MM}_{142}\right)$ [24].

Table 1 compares the clinical TSE status, based on the short clinical assessment, with the pathological TSE

Table 1 Comparison of clinical and pathological TSE status in the herd cull based on short clinical assessment

\begin{tabular}{|c|c|c|c|c|}
\hline & & Pathological & TSE status & \\
\hline Herd & Clinical TSE status & $\begin{array}{l}\text { Positive } \\
\text { (brain) }\end{array}$ & $\begin{array}{l}\text { Positive } \\
\text { (LRS only) }\end{array}$ & $\begin{array}{l}\text { Negative } \\
\text { (brain \& LRS) }\end{array}$ \\
\hline \multirow[t]{3}{*}{ A } & No clinical signs & $4^{a}$ & 2 & 129 \\
\hline & Inconclusive & $1^{b}$ & 0 & 14 \\
\hline & Scrapie suspect & 0 & 0 & 0 \\
\hline \multirow[t]{3}{*}{$B$} & No clinical signs & 29 & 28 & 109 \\
\hline & Inconclusive & 5 & 4 & 16 \\
\hline & Scrapie suspect & 3 & 1 & 1 \\
\hline
\end{tabular}

${ }^{a}$ Includes three animals without detectable PrPd in LRS.

${ }^{b}$ No PrPd in LRS detected. status, based on $\operatorname{PrP}^{\mathrm{d}}$ detection in tissues of the LRS and brain, for each herd cull. The test sensitivity (scrapie suspect $=$ TSE positive, all others $=$ TSE-negative) based on the short clinical assessment was 3.9\% but increased to $11.7 \%$ if inconclusive cases were also considered to be TSE-positive (ignoring goats that were difficult to milk since it would not have been detected during the assessment). Specificity was $99.6 \%$ and $88.5 \%$ respectively. Of the clinically unremarkable goats of herd B based on the short clinical assessment, four scrapie-affected goats (two each positive in brain and LRS only) and six scrapie-negative animals were difficult to milk (see additional file 3: 'G08-1349 milking' showing a scrapie-affected goat that is difficult to milk).

The percentage of animals that displayed selected clinical signs at the short clinical assessment is shown in Table 2. Only three signs were significantly more frequently displayed in TSE-positive animals, which were all associated with pruritus. Specific and localised skin abnormalities included scales and crusts on the pinnae of one goat and ring-shaped hair loss with central erythema in two goats. Lice were found in the coat of one goat. None of these goats displayed a positive scratch test and all were scrapie-negative.

The single CNS-positive goat with inconclusive signs of scrapie in herd A (see Table 1) had a bilaterally absent menace response. Three of the four scrapie-affected goats in herd B had a positive scratch test, one of which had $\operatorname{PrP}^{\mathrm{d}}$ only in LRS, whereas the fourth was ataxic with a head tremor and extensive hair loss with skin lesions (see additional file 4: 'G08-1407 tremor' showing head tremor and hair loss). A positive scratch test was also displayed by one TSE-negative goat. Of the nine TSE-positive goats in herd $B$, which had inconclusive signs at the short assessment, two were ataxic (one with $\operatorname{PrP}^{\mathrm{d}}$ in LRS only) and seven displayed a tremor (three with $\operatorname{PrP}^{\mathrm{d}}$ in LRS only). The 16 TSE-negative goats with inconclusive signs of scrapie displayed a head, body or leg tremor (11 goats), ataxia (two goats) or an inconclusive scratch test response (three goats: crouching or inconsistent head/lip movements when scratched).

Description of the signs in goats monitored in more detail

Of the 24 goats from herd B that were kept for monitoring of clinical progression or milk collection, eight were scrapie-negative on postmortem tests: four continued to show no apparent signs of scrapie and four showed inconclusive signs prior to cull (see Table 3 ). The remaining 16 scrapie-infected goats (see Table 4) were or became clinical suspects on or after arrival at VLA (six goats, also included in. Figure 1), developed inconclusive signs of scrapie (seven goats) or continued to show no apparent signs of scrapie prior to cull (three goats).

Figure 1 summarises the progression of seven selected clinical signs in seven scrapie-affected goats, one of 
Table 2 Individual signs in $\mathbf{3 4 6}$ goats assessed by the short protocol

\begin{tabular}{|c|c|c|c|c|}
\hline Postmortem TSE status: CLINICAL SIGN & $\mathrm{CNS}+(\mathrm{N}=42)$ & LRS $+(\mathrm{N}=35)$ & Negative $(\mathrm{N}=269)$ & p-value \\
\hline \multicolumn{5}{|l|}{ Abnormalities in behaviour } \\
\hline \multicolumn{5}{|l|}{ Behaviour when handled } \\
\hline - Nervous/went down & $4.8 \%$ & $8.6 \%$ & $10.0 \%$ & ns \\
\hline - Dull/docile & $0 \%$ & $0 \%$ & $2.2 \%$ & ns \\
\hline Spontaneous startle & $2.4 \%$ & $0 \%$ & $0 \%$ & ns \\
\hline Teeth grinding & $2.4 \%$ & $0 \%$ & $0.4 \%$ & ns \\
\hline \multicolumn{5}{|l|}{ Abnormalities in sensation } \\
\hline \multicolumn{5}{|l|}{ Hand clap ${ }^{1}$} \\
\hline - Startle once & $2.4 \%$ & $0 \%$ & $0.7 \%$ & ns \\
\hline - Startle more than twice & $2.4 \%$ & $0 \%$ & $1.5 \%$ & ns \\
\hline \multicolumn{5}{|l|}{ Scratch test } \\
\hline - Positive & $4.8 \%$ & $2.9 \%$ & $0.4 \%$ & 0.04 \\
\hline - Unable to test & $0 \%$ & $11.4 \%$ & $4.1 \%$ & ns \\
\hline - Inconclusive & $0 \%$ & $0 \%$ & $2.2 \%$ & ns \\
\hline Hair loss & $54.8 \%$ & $57.1 \%$ & $42.0 \%$ & 0.04 \\
\hline Poll & $45.2 \%$ & $42.9 \%$ & $23.0 \%$ & 0.0005 \\
\hline Lateral neck & $9.5 \%$ & $5.7 \%$ & $6.7 \%$ & ns \\
\hline Base of/dorsal neck & $9.5 \%$ & $5.7 \%$ & $6.7 \%$ & ns \\
\hline Nose & $7.1 \%$ & $2.9 \%$ & $7.1 \%$ & ns \\
\hline Eyelid & $2.4 \%$ & $2.9 \%$ & $1.1 \%$ & ns \\
\hline Side abdomen/flank/chest & $0 \%$ & $2.9 \%$ & $2.6 \%$ & ns \\
\hline Shoulder & $2.4 \%$ & $0 \%$ & $4.1 \%$ & ns \\
\hline Back/tail base & $2.4 \%$ & $0 \%$ & $3.3 \%$ & ns \\
\hline Rump & $2.4 \%$ & $0 \%$ & $2.6 \%$ & ns \\
\hline Leg & $2.4 \%$ & $0 \%$ & $0.7 \%$ & ns \\
\hline Skin abnormalities & $26.2 \%$ & $28.6 \%$ & $12.3 \%$ & 0.002 \\
\hline Hair short & $19.0 \%$ & $8.6 \%$ & $27.9 \%$ & 0.02 \\
\hline Lateral neck & $11.9 \%$ & $2.9 \%$ & $18.6 \%$ & 0.02 \\
\hline Base of/dorsal neck & $4.8 \%$ & $0 \%$ & $1.1 \%$ & ns \\
\hline Back/tail base & $2.4 \%$ & $0 \%$ & $3.3 \%$ & ns \\
\hline Shoulder & $2.4 \%$ & $0 \%$ & $4.8 \%$ & ns \\
\hline Poll & $0 \%$ & $2.9 \%$ & $0.4 \%$ & ns \\
\hline Nose & $0 \%$ & $2.9 \%$ & $1.9 \%$ & ns \\
\hline Rump & $0 \%$ & $0 \%$ & $1.5 \%$ & ns \\
\hline Eyelid & $0 \%$ & $0 \%$ & $0.4 \%$ & ns \\
\hline Side abdomen/flank & $0 \%$ & $0 \%$ & $0.4 \%$ & ns \\
\hline \multicolumn{5}{|l|}{ Menace response $^{1}$} \\
\hline - Exaggerated & $4.8 \%$ & $2.9 \%$ & $4.5 \%$ & ns \\
\hline - Absent & $2.4 \%$ & $0 \%$ & $2.2 \%$ & ns \\
\hline \multicolumn{5}{|l|}{ Abnormalities in posture and movement } \\
\hline Ataxia/hypermetria ${ }^{2}$ & $4.8 \%$ & $2.9 \%$ & $0.7 \%$ & ns \\
\hline Abnormal posture & $2.4 \%$ & $2.9 \%$ & $1.5 \%$ & ns \\
\hline \multicolumn{5}{|l|}{ Tremor } \\
\hline - Head/body & $7.1 \%$ & $5.7 \%$ & $6.3 \%$ & ns \\
\hline - Limbs & $5.0 \%$ & $2.9 \%$ & $0.7 \%$ & ns \\
\hline \multicolumn{5}{|l|}{ Other signs } \\
\hline$\overline{\text { BCS poor }(\leq 2)^{3}}$ & $4.8 \%$ & $2.9 \%$ & $22.8 \%$ & 0.0001 \\
\hline
\end{tabular}

Only tested in 344 goats $^{1}, 345$ goats $^{2}, 342$ goats $^{3}$.

$n s=$ not significant $(p>0.5)$. 
Table 3 Signs of scrapie-negative goats kept for milk collection or monitoring of clinical progression

\begin{tabular}{|c|c|c|c|c|c|}
\hline Animal & Days at VLA & Genotype $_{127-142-211-222-240}$ & Breed & Clinical status \& signs at arrival & Clinical status \& signs prior to cull \\
\hline G08-1136 & 38 & GIRQP/GMRQP & AN & No TSE signs (difficult to milk)* & No TSE signs ${ }^{1,4}$ \\
\hline G08-1376 & 38 & GIRQP/GIRQP & AN & No TSE signs (difficult to milk)* & No TSE signs ${ }^{1,4}$ \\
\hline G08-1382 & 39 & GIRQP/GMRQP & AN & No TSE signs (difficult to milk)* & No TSE signs ${ }^{1,4}$ \\
\hline G08-1122 & 65 & GIRQP/GMRQP & AN & No TSE signs (difficult to milk)* & No TSE signs ${ }^{2-4}$ \\
\hline G08-1342 & 25 & GIRQP/GIRQP & AN & No TSE signs (difficult to milk)* & $\begin{array}{l}\text { Inconclusive (kicking and lying down } \\
\text { at milking) }\end{array}$ \\
\hline G08-1454 & 37 & GIRQP/GMRQP & SA & No TSE signs (difficult to milk)* & Inconclusive (leg tremor) $)^{1,4}$ \\
\hline G08-1115 & 65 & GMRQP/GMRQP & AN & $\begin{array}{l}\text { Inconclusive (head and body tremor, } \\
\text { teeth grinding) }\end{array}$ & $\begin{array}{l}\text { Inconclusive [body tremor, } \\
\text { inconclusive scratch test (crouching), } \\
\text { also diarrhoea] }\end{array}$ \\
\hline G08-1144 & 39 & GMRQP/GMRQP & SA & $\begin{array}{l}\text { Scrapie (positive scratch test, } \\
\text { repeated startle to auditory stimuli) }\end{array}$ & $\begin{array}{l}\text { Inconclusive (pruritic behaviour on } \\
\text { camera recordings but negative } \\
\text { scratch test })^{1,3}\end{array}$ \\
\hline
\end{tabular}

AN = Anglo-Nubian, SA = Saanen.

The clinical TSE status on arrival was assessed using the short protocol; for the assessment of the clinical TSE status prior to cull clinical assessments (short ${ }^{1}$ or long $^{2}$ protocol), CCTV observations ${ }^{3}$ and behaviour at milking ${ }^{4}$ were taken into consideration.

* Inconclusive signs if behaviour during milking was included.

which (G08-1430) presented with $\operatorname{PrP}^{\mathrm{d}}$ in LRS only. This goat displayed a positive scratch test, which could be elicited by merely applying pressure to the spine prior to cull (see additional file 5: 'G08-1430 scratch test' showing the response of this goat to the scratch test).

Abnormalities in behaviour and sensation Entering the pen and subsequent movement of animals resulted in sudden collapse of G08-1447 into lateral recumbency where it remained motionless until it was touched.

Kicking out or restless behaviour when the hind legs were touched, which in extreme cases culminated in the animal going down on the floor, was observed in four of eight CNS-positive and one of five LRS only-positive scrapie cases prior to cull. It was not seen in two TSEnegative animals prior to cull.

Of the 24 goats from herd B, 18 (seven TSE-negative, seven CNS-positive and four LRS only-positive) displayed hair loss prior to cull. Various areas were affected, but most frequently the poll (13 goats: seven TSE-negative, four CNS-positive and two LRS only-positive) and neck (five goats: two TSE-negative, two CNSpositive and one LRS only-positive).

The pruritic behaviour observed on camera recordings of one reported scrapie suspect of herd $\mathrm{A}$ and 17 goats of herd B is shown in Table 5. Nibbling was on average twice as frequent as scratching or rubbing of body parts. Abnormalities in posture and movement The camera recordings of the 17 goats of herd B (four TSE-negative, five LRS only-positive and eight CNS-positive goats) demonstrated abnormal rising with the fore limbs first (four scrapie-affected goats, three of which were positive on LRS only), which was not always consistently observed (see additional file 6: 'G08-1389 getting up' showing a scrapie-affected goat with pruritic behaviour rising normally and abnormally) and delayed lying down (not immediately flexing the hind limbs to lie down; seven scrapie-affected animals, two of which were positive on LRS only; three were goats that also got up abnormally).

Proprioceptive deficits based on slow limb correction were suspected in one goat (G08-1475).

\section{Goats reported as scrapie suspects}

All five goats had detectable $\operatorname{PrP}^{\mathrm{d}}$ in the brain and LRS (palatine tonsil 5/5, RAMALT 4/5). The clinical signs are displayed in Table 6 (four goats, see additional file 7: 'G08-1110 startle' showing repeated spontaneous startle) and Figure 1 (one goat: GZ1100, four year-old Saanen doe with clinical onset 80 days prior to arrival at VLA, Genotype: GIRQP/GIRQP).

Abnormalities in behaviour and sensation Touching of the hind limbs during testing of proprioceptive deficits elicited kicking or restless movement in four of the five goats, including the goat kept for monitoring of clinical progression (GZ1100), although it had not been observed at the first examination.

Hair loss was present in four goats involving various areas, including the neck (G08-1111-1113, herd B) and poll (G08-1110, G08-1112) or the shoulder and caudal back (G08-1153, herd A).

Abnormalities in posture and movement Postural abnormalities included a wide-based stance of the hind limbs, standing with the neck extended or with the head lowered and the hind limbs camped back. Hypermetria in addition to ataxia was displayed by three goats (see additional file 8: 'G08-1111 gait' showing an ataxic, wide-based gait with occasional high stepping of the fore limbs).

Hind limb correction after pushing the animal to one side appeared to be abnormal in two goats, with 
Table 4 Signs of scrapie-affected goats kept for milk collection or monitoring of clinical progression

\begin{tabular}{|c|c|c|c|c|c|}
\hline Animal & $\begin{array}{l}\text { Days } \\
\text { at } \\
\text { VLA }\end{array}$ & $\begin{array}{l}\text { Genotype } \\
127-142-211-222-240\end{array}$ & Breed & $\begin{array}{l}\text { Clinical status \& signs at } \\
\text { arrival }\end{array}$ & Clinical status \& signs prior to cull \\
\hline \multicolumn{6}{|c|}{ Scrapie positive (LRS only) } \\
\hline G08-1472 & 37 & GIRQP/GIRQP & AN & $\begin{array}{l}\text { No signs of TSE (difficult to milk) } \\
*\end{array}$ & No signs of TSE ${ }^{1,4}$ \\
\hline G08-1427 & 86 & $\mathrm{XIRQP} / \mathrm{XIRQP}$ & AN & No signs of TSE & No signs of TSE 2,4 \\
\hline G08-1396 & 107 & GIRQS/GIRQP & AN & No signs of TSE & Scrapie (see Fig. 1), pruritic activity on camera recordings $s^{2,3}$ \\
\hline G08-1143 & 99 & GIRQS/GIRQS & AN & Inconclusive (ataxia) & Inconclusive (pruritic behaviour on camera recordings) ${ }^{2-4}$ \\
\hline G08-1389 & 107 & GIRQP/GIRQP & SA & No signs of TSE & $\begin{array}{l}\text { Inconclusive (self-induced scratch reflex, pruritic behaviour and } \\
\text { abnormal rising on camera recordings), }\end{array}$ \\
\hline G08-1465 & 38 & GIRQP/GMRQP & AN & $\begin{array}{l}\text { No signs of TSE (difficult to milk) } \\
*\end{array}$ & Inconclusive (difficult to milk (kicking) ${ }^{1,4}$ \\
\hline G08-1430 & 106 & GIRQP/GIRQP & AN & Scrapie (positive scratch test) & Scrapie (see Fig. 1) ) $^{2,3}$ \\
\hline \multicolumn{6}{|c|}{ Scrapie positive (CNS and LRS) } \\
\hline G08-1451 & 98 & GIRQP/GMRQP & AN & No signs of TSE & $\begin{array}{l}\text { Inconclusive (pruritic behaviour and abnormal rising on camera } \\
\text { recordings) }\end{array}$ \\
\hline G08-1415 & 98 & GIRQS/GMRQP & TG & No signs of TSE & $\begin{array}{l}\text { Inconclusive (prefers to stay close to pen, kicking when hind } \\
\text { limbs touched, head tremor at times, hair loss on left flank not } \\
\text { previously seen, pruritic activity on camera recordings) }\end{array}$ \\
\hline G08-1383 & 99 & GIRQS/GMRQP & SA & No signs of TSE & Inconclusive (inconclusive scratch test) ${ }^{2-4}$ \\
\hline G08-1365 & 107 & GIRQP/GMRQP & SA & No signs of TSE & Inconclusive (inconclusive scratch test) ${ }^{2,3}$ \\
\hline G08-1475 & 86 & GIRQP/GIRQP & AN & $\begin{array}{l}\text { No signs of TSE (difficult to milk) } \\
*\end{array}$ & Scrapie (see Fig. 1), difficult to milk (kicking) ${ }^{2-4}$ \\
\hline G08-1373 & 107 & $\mathrm{SIRQP} / \mathrm{GIRQP}$ & SA & $\begin{array}{l}\text { Inconclusive (head and body } \\
\text { tremor) }\end{array}$ & Scrapie (see Fig. 1) $)^{2,3}$ \\
\hline G08-1447 & 57 & GIRQP/GIRQP & AN & $\begin{array}{l}\text { Inconclusive (head tremor, } \\
\text { nervous on head tests) }\end{array}$ & $\begin{array}{l}\text { Scrapie (see Fig. 1), difficult to milk (kicking, sitting down), pruritic } \\
\text { activity on camera recordings }\end{array}$ \\
\hline G08-1340 & 39 & GIRQP/GMRQP & AN & $\begin{array}{l}\text { Scrapie (positive scratch test, } \\
\text { teeth grinding) }\end{array}$ & No signs of TSE (negative scratch test) ${ }^{1}$ \\
\hline G08-1460 & 98 & GIRQP/GIRQP & AN & $\begin{array}{l}\text { Scrapie (positive scratch test, } \\
\text { repeated startle to auditory } \\
\text { stimuli) }\end{array}$ & Scrapie (see Fig. 1) \\
\hline
\end{tabular}

AN = Anglo-Nubian, SA = Saanen, TG = Toggenburg.

The clinical TSE status on arrival was assessed using the short protocol; for the assessment of the clinical TSE status prior to cull clinical assessments (short ${ }^{1}$ or long $^{2}$ protocol), camera recordings ${ }^{3}$ and behaviour at milking ${ }^{4}$ were taken into consideration.

* Inconclusive signs if behaviour during milking was included.

crouching (G08-1110) and simultaneous hopping with both hind legs (G08-1111).

Other signs Blindfolding resulted in circling in an anticlockwise direction in goat GZ1100, which had not been observed three weeks earlier.

Drooling of saliva was exhibited by goat G08-1112, which also presented with dyspnoea, increased respiratory noise and halitosis suggestive of aspiration pneumonia, which was confirmed histopathologically. This goat was also the only animal with spontaneous teeth grinding.

\section{Association of clinical signs with $\operatorname{PrP}^{\mathrm{d}}$ accumulation in the brain}

The magnitude of $\operatorname{PrP}^{\mathrm{d}}$ accumulation in the thalamus with the corresponding pruritic activity is shown in Table 5. Goats that presented with a similarly high thalamic score as G08-1447 and G081475 but were only examined by the short protocol were G08-1407 (score: 2.5; negative scratch test but extensive hair loss, see additional file 4: 'G08-1407 tremor' demonstrating hair loss) and G08-1469, which was clinically unremarkable without any hair loss.

Of the 37 CNS-positive goats in herd B, twelve presented with $\operatorname{PrP}^{\mathrm{d}}$ accumulation in the cerebellum with the following scores and animals in parentheses: 0.2 (one without any signs), 0.5 (five, including G08-1460; two without any signs), 1 (two, including G08-1373, one without any signs), 1.5, 2, 2.5 and 3 (one each, including G08-1407, G08-1447 and G08-1475; one without any 


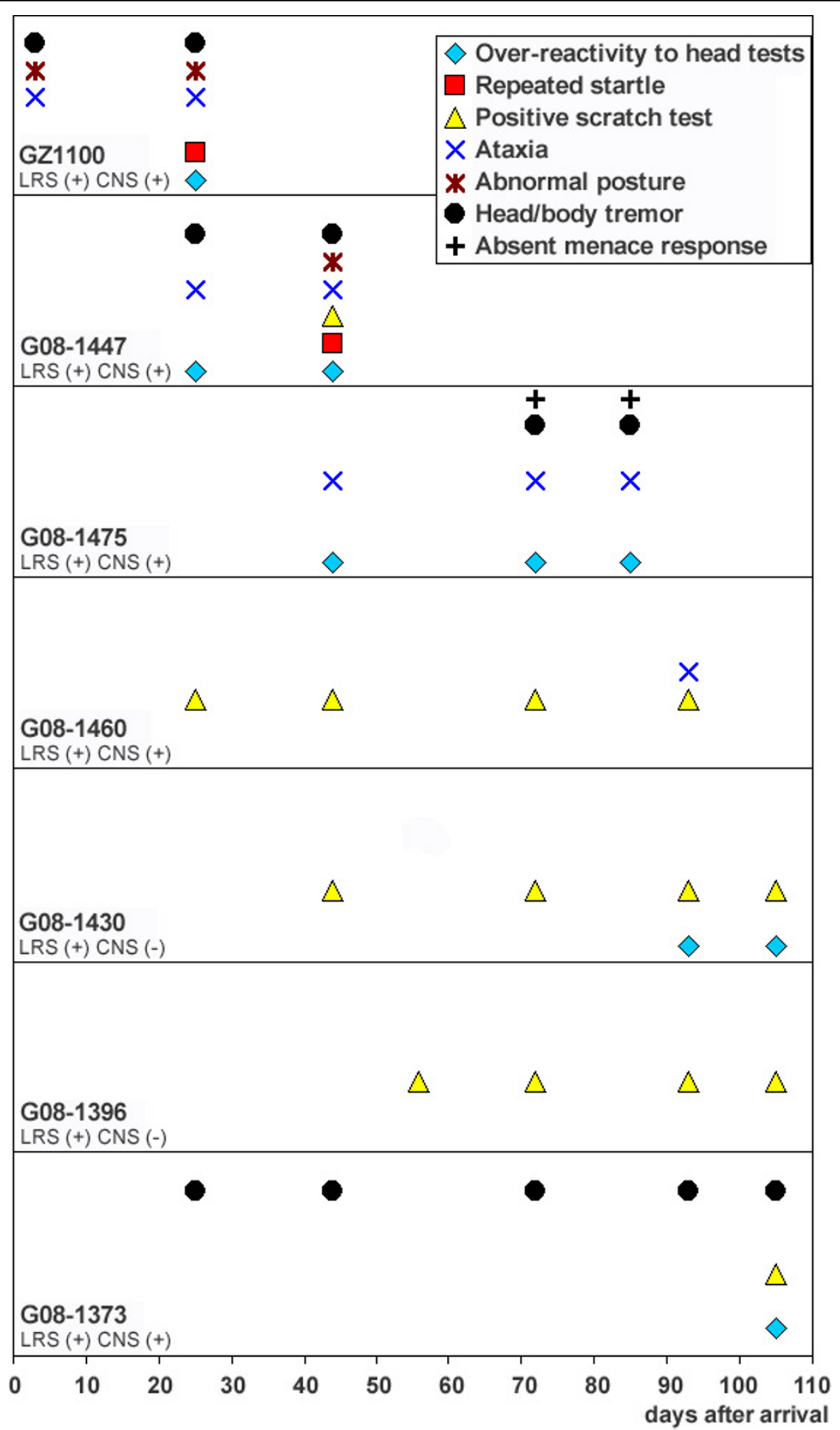

Figure 1 Timeline of clinical progression in scrapie-affected goats. Each column of symbols for one animal represents a time point when an examination was carried out in this particular animal. Days after arrival refers to the days after transport to VLA Weybridge. Only the first animal (GZ1100, herd A) was a clinical suspect reported by the farmer whereas the other goats arrived without clinical suspicion as part of the herd cull (herd B). The latter goats displayed no apparent signs (G08-1475, G08-1396) or inconclusive signs of scrapie (G08-1447: head tremor, over-reactivity to handling, G08-1373: hind limb tremor) or were considered scrapie suspects [G08-1460: positive scratch test, repeated overreactivity to auditory stimuli (hand clapping), G08-1430: positive scratch test] based on the short clinical assessment. 
Table 5 Pruritic behaviour observed on camera recordings

\begin{tabular}{|c|c|c|c|c|c|c|}
\hline Animal No & Total recorded time [min] & Pruritus score & Rubbing & Nibbling & Scratching & PrPd $^{d}$ score (thalamus) \\
\hline \multicolumn{7}{|c|}{ Scrapie negative (LRS \& CNS) } \\
\hline G08-1144 & 71 & 1.00 & $24 \%$ & $55 \%$ & $21 \%$ & 0 \\
\hline G08-1122 & 120 & 0.24 & $17 \%$ & $62 \%$ & $21 \%$ & 0 \\
\hline G08-1342 & 54 & 0.22 & $0 \%$ & $83 \%$ & $17 \%$ & 0 \\
\hline G08-1115 & 120 & 0.18 & $43 \%$ & $38 \%$ & $19 \%$ & 0 \\
\hline \multicolumn{7}{|c|}{ Scrapie positive (LRS Only) } \\
\hline G08-1389 & 1928 & 0.78 & $20 \%$ & $59 \%$ & $21 \%$ & 0 \\
\hline G08-1143 & 224 & 0.64 & $19 \%$ & $68 \%$ & $13 \%$ & 0 \\
\hline G08-1396 & 2019 & 0.60 & $20 \%$ & $47 \%$ & $33 \%$ & 0 \\
\hline G08-1430 & 1712 & 0.49 & $46 \%$ & $50 \%$ & $4 \%$ & 0 \\
\hline G08-1427 & 80 & 0.03 & $0 \%$ & $100 \%$ & $0 \%$ & 0 \\
\hline \multicolumn{7}{|c|}{ Scrapie positive (CNS \& LRS) } \\
\hline G08-1447 & 298 & 0.64 & $48 \%$ & $30 \%$ & $22 \%$ & 3.0 \\
\hline G08-1415 & 785 & 0.50 & $27 \%$ & $57 \%$ & $16 \%$ & 0 \\
\hline G08-1460 & 199 & 0.47 & $16 \%$ & $68 \%$ & $16 \%$ & 0 \\
\hline G08-1373 & 654 & 0.45 & $45 \%$ & $34 \%$ & $21 \%$ & 1.0 \\
\hline G08-1365 & 1828 & 0.44 & $18 \%$ & $57 \%$ & $25 \%$ & 0 \\
\hline G08-1475 & 236 & 0.41 & $37 \%$ & $56 \%$ & $7 \%$ & 3.0 \\
\hline G08-1383 & 609 & 0.39 & $26 \%$ & $49 \%$ & $25 \%$ & 0 \\
\hline G08-1451 & 725 & 0.37 & $12 \%$ & $69 \%$ & $19 \%$ & 0.5 \\
\hline G08-1153* & 107 & 0.32 & $6 \%$ & $35 \%$ & $59 \%$ & $\overline{N / D}$ \\
\hline$G Z 1100 * \$$ & 120 & 0.31 & $22 \%$ & $71 \%$ & $24 \%$ & $\mathrm{~N} / \mathrm{D}$ \\
\hline
\end{tabular}

Animal numbers in bold font: Positive scratch test prior to cull.

* Clinical suspect from herd A reported by the farmer; all others are from herd B; a pruritic score $\geq 0.5$ (underlined font) was considered excessive.

${ }^{\$}$ Fleas found in coat prior to cull.

$\mathrm{N} / \mathrm{D}=$ Not determined.

Table 6 Clinical onset and signs in four scrapie suspects reported by the farmer

\begin{tabular}{llll}
\hline Animal No & G08-1110 & G08-1111 & G08-1112 \\
\hline Breed & Anglo-Nubian & Anglo-Nubian & Saanen \\
\hline Age [years] & 3 & 3 & 3 \\
\hline Genotype 127-142-211-222-240 & GIRQP/GIRQP & GIRQP/GIRQS & GIRQP/GIRQP \\
\hline Clinical onset prior to arrival at VLA & 6 days ago & 6 days ago & 6 days ago \\
\hline Over-reactivity head tests & + & - & - \\
\hline Repeated startle & + & - & - \\
\hline Positive scratch test & + & - & - \\
\hline Ataxia & + & + & + \\
\hline Abnormal posture & + & + & + \\
\hline Head/body tremor & + & + & + \\
\hline Absent menace response & - & + & + \\
\hline+ sign present. & + & + \\
\hline
\end{tabular}

\footnotetext{
- sign absent.
}

signs). G08-1475 had the highest score and displayed signs most highly suggestive of cerebellar dysfunction (general ataxia with forelimb hypermetria, head tremor, absent menace response).

Table 7 compares the clinical status prior to cull of the CNS-positive goats in herd B, taking into account all assessments, with the total score of $\operatorname{PrP}^{\mathrm{d}}$ accumulation representative of the severity of $\operatorname{PrP}^{\mathrm{d}}$ accumulation in the brain. The score of those goats that were retained for longer and thus subject to a more detailed clinical assessment is given in the examples. 
Experimentally infected cases (BSE)

All animals presented with $\operatorname{PrP}^{\mathrm{d}}$ in the brain; the absence of intraneuronal immunolabelling in the obex with antibody $\mathrm{P} 4$ was indicative of $\mathrm{BSE}$. $\mathrm{PrP}^{\mathrm{d}}$ immunolabelling was also observed in some LRS tissue (medial retropharyngeal lymph node $4 / 5$, mesenteric lymph node $1 / 5$, palatine tonsil $3 / 5$, RAMALT $0 / 5$ ).

Table 8 lists the incubation period and duration of clinical disease. The progression of seven selected clinical signs over the course of the disease is displayed in Figure 2.

\section{Abnormalities in behaviour and sensation}

Dullness, characterised by a low head carriage when undisturbed, and inappetence were only observed in one goat (GX1030).

Teeth grinding was displayed by three goats during the incubation period but only two goats presented with this sign at the examination prior to cull (one during head tests and one spontaneously).

Curious behaviour towards cranial nerve assessment, characterised by sniffing and nibbling of animal handlers and instruments, gave way to increasingly aversive behaviour, such as head twisting and tossing or jerky head movements. This over-reactivity to head tests became eventually so extreme that - with the exception of goat GX1030 - the assessment had to be abandoned (see additional file 9: 'GX1032 menace' showing the difficulty in holding the head of this goat). Head tossing or shaking was also observed during the gait assessment in a corridor and - in three goats - occasionally on camera recordings made prior to cull. Similarly, goats developed over-reactivity to touch of the hind limbs, characterised

Table 7 Comparison of total brain $\operatorname{PrP}^{\mathrm{d}}$ score with clinical TSE status

\begin{tabular}{lll}
\hline $\begin{array}{l}\text { Clinical status } \\
\text { prior to cull }\end{array}$ & $\begin{array}{l}\text { Mean total } \\
\text { PrP }^{\mathbf{d}} \text { score in } \\
\text { brain (range) }\end{array}$ & $\begin{array}{l}\text { Examples (goats monitored } \\
\text { for clinical progression) }\end{array}$ \\
\hline $\begin{array}{l}\text { No signs of TSE } \\
(\mathrm{N}=25)\end{array}$ & $3.3(0.2-20)$ & G08-1430: 0.5 \\
\hline $\begin{array}{l}\text { Inconclusive } \\
(N=7)\end{array}$ & $4.3(0.5-10.4)$ & $\begin{array}{l}\text { G08-1365, G08-1383: 2.5; } \\
\text { G08-1415: 3.5; G08-1451: } 4\end{array}$ \\
\hline $\begin{array}{l}\text { Scrapie suspect } \\
(N=5)\end{array}$ & $17.2(7-27.5)$ & G08-1460: 7; G08-1373: 12; \\
\hline
\end{tabular}

by kicking when the legs were touched for tests of proprioception (all goats) or by exaggerated responses to withdrawal reflex testing of the hind limbs (two goats). Three goats developed an absent menace response, which was unilateral in one animal (GX1035) - see additional file 9: 'GX1032 menace' showing this goat with an absent menace response. Other tests of cranial nerve function and ophthalmoscopic examination - if tolerated by the animals - and assessment of tail and anal tone did not reveal any abnormalities.

The scratch test was positive in three animals at the last examination prior to cull, one of which (GX1031) responded even to mere pressure on the spine (see additional file 10: 'GX1031 scratch test' with this goat responding to scratching of the dorsum). Two goats with a positive scratch test prior to cull developed localised hair loss (base of neck, above shoulders and poll, left eyelid retrospectively) and had shorter hair (rump and lateral neck, retrospectively). Based on the last camera observations prior to cull (see Figure 2 for the time point during the incubation period), only GX1035 was considered to be excessively pruritic (pruritus score: 0.55; GX1030: 0.07, GX1034: 0.13, GX1032: 0.15, GX1031: 0.31).

\section{Abnormalities in posture and movement}

Goats predominantly presented with hypermetria except for the dull goat GX1030, which developed a stiff, hypometric gait.

Postural reactions tested by excessively abducting or adducting limbs appeared to be slow in the hind limbs at some stage during the incubation period but this was not always consistently observed on subsequent examinations and this test was later impossible to evaluate because of kicking. The limited usefulness of this test in large animals has been reported [25]. Hind limb correction after pushing the animal to one side appeared to be slow in two goats at the last examination prior to cull.

Whilst none of the goats were observed to rise abnormally with their forelimbs first, three goats laid down slowly according to the last camera observations prior to cull (see additional file 11: 'GX1030 lying down' showing this goat's hesitation in lying down).

\section{Other signs}

Weight loss prior to cull was recorded for four goats (GX1030: 14.5\%, GX1032: 14\%, GX1035: 12.7\%,

Table 8 Genotype and clinical parameters in BSE-infected goats

\begin{tabular}{lllll}
\hline Animal & Genotype & Clinical onset [mpi] & Cull date [mpi] \\
\hline GX1030 & GIRQ-211-222-240 & Clinical duration [d] & 30.5 & 199 \\
\hline GX1031 & GIRQP/GMRQP & 24 & 34 & 140 \\
\hline GX1032 & GMRQP/GMRQP & 29.5 & 26.5 & 176 \\
\hline GX1034 & GMRQP/GMRQP & 20.5 & 25 & 41 \\
\hline GX1035 & GMRQP/GMRQP & 24 & 25 & 39 \\
\hline
\end{tabular}




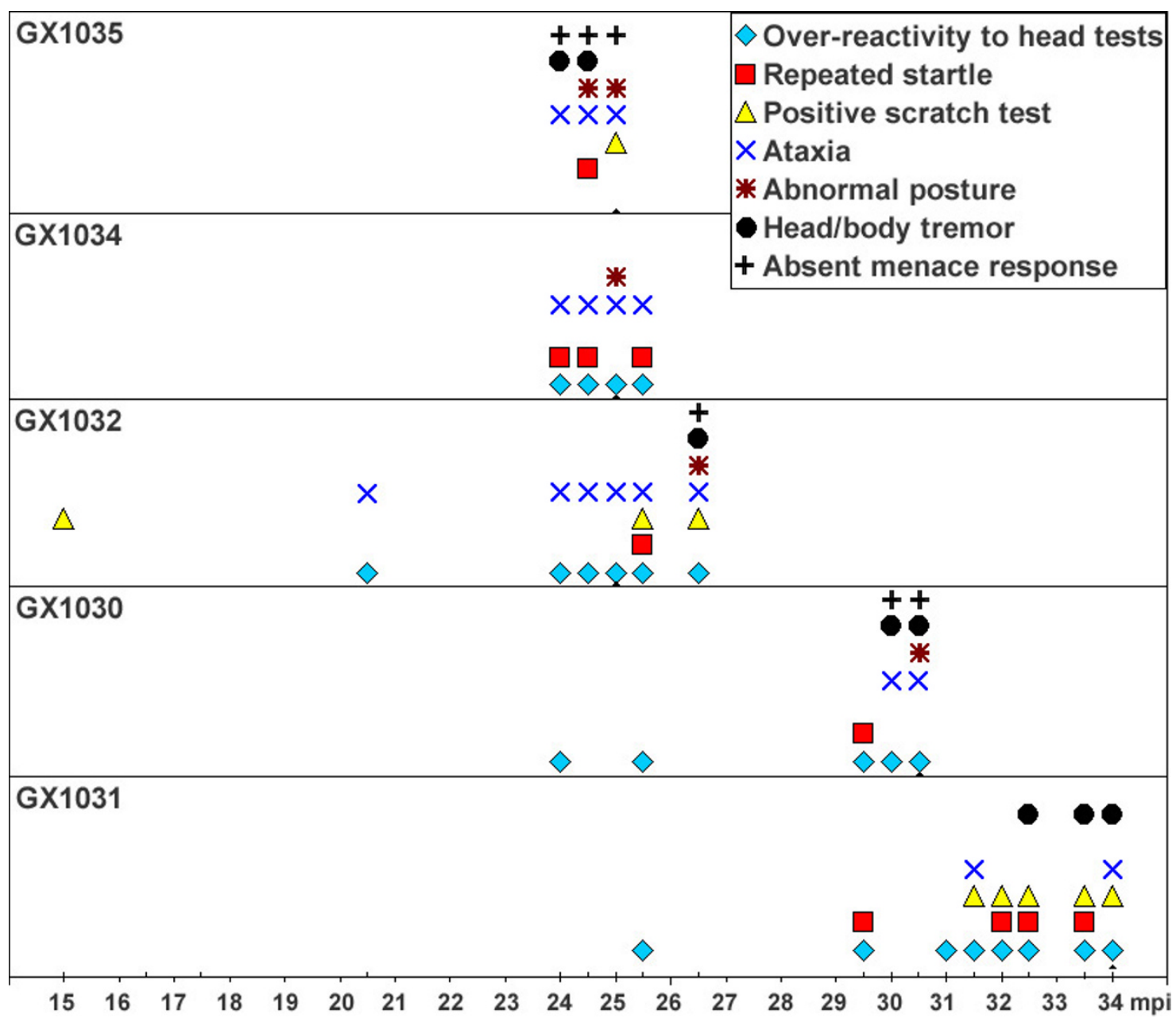

Figure 2 Timeline of clinical progression in BSE-infected goats. Tick marks on the $x$-axis that protrude inside the box represent time points when neurological examinations were carried out. A small black triangle ( $\mathbf{\Delta})$ indicates the time when the last CCTV observation for a particular animal was available. None of the signs were displayed at the pre-inoculation examination.

GX1034: $8.4 \%$ of its previous body weight) but the duration of the progressive weight loss varied (GX1030:14, GX1032: 130, GX1035: 66, GX1034: 58 days between the last day of recorded weight gain and the day of last weighing prior to cull).

\section{Discussion}

As scrapie is a neurological disease the clinical diagnosis should be based on a neurological examination. This is, however, time-consuming and not applicable when many animals have to be examined, e.g. at abattoirs. The short assessment protocol was designed to evaluate signs usually associated with classical or atypical scrapie in small ruminants, such as nervousness, pruritus, tremor, ataxia and poor bodily condition. The signs selected for identification of suspect cases (tremor, positive scratch test, extensive hair loss, ataxia and absent menace response) were highly specific but most of the scrapie cases would not have been detected using these criteria. This was to be expected since it has been shown previously that the postmortem tests are superior to a clinical examination of sheep in scrapie-affected flocks, even if limited to brain examination only [26]. The conduction of the short clinical assessment lasted approximately five minutes per animal. After taking into account the signs that were significantly more frequent in confirmed scrapie cases in the herd cull (see Table 2) or seen at clinical end-stage in BSE or scrapie, it appears to be sufficient to assess animals for postural and gait abnormalities, signs of pruritus (scratch testing, hair loss predominantly on the poll and neck) and visual impairment (menace response testing).

As reported previously for herd B [24], evident (definite) signs of scrapie were found exclusively in goats homozygous for isoleucine at codon 142 of the caprine PRNP although one heterozygous $\left(\mathrm{IM}_{142}\right)$ goat was 
initially considered to be a clinical suspect but the signs later regressed. Equally, all five clinical suspects reported by the farmer were $\mathrm{I}_{142}$ homozygotes, which suggests that scrapie in $\mathrm{IM}_{142}$ or $\mathrm{MM}_{142}$ goats may cause subclinical or a more protracted disease. BSE caused clinical disease in these PRNP genotypes but this may be due to the intracerebral route of infection. Although scrapie was diagnosed in goats of all breeds, clinical disease was not observed in the Alpine breed, but the number of clinically affected animals is too small to draw any conclusion on the effect of breed on the clinical presentation.

Contrary to TSE in cattle, where $\operatorname{Pr} \mathrm{P}^{\mathrm{d}}$ detection is almost entirely restricted to the CNS, small ruminants may present with $\mathrm{PrP}^{\mathrm{d}}$ in tissues of the LRS, some of which (tonsil, nictitating membrane and RAMALT) are accessible in the live animal. RAMALT samples, examined from goats in herd A (GZ1100 [18]), herd B [24] and BSE-affected goats did not always present with detectable $\operatorname{PrP}^{\mathrm{d}}$ despite $\operatorname{PrP}^{\mathrm{d}}$ accumulation in other tissues of the LRS. There is currently no evidence that $\operatorname{PrP}^{\mathrm{d}}$ accumulation in LRS produces clinical abnormalities since antemortem tests generally detect pre-clinical scrapie cases $[27,28]$, hence any animal with detectable $\operatorname{PrP}^{\mathrm{d}}$ in LRS only (35 cases in the present study) was likely to be missed as scrapie suspect. Two animals with detectable $\operatorname{PrP}^{\mathrm{d}}$ restricted to the LRS, however, displayed signs of scrapie. There are no pathognomonic clinical signs for scrapie (scrapie-like signs were also observed in goats without detectable $\operatorname{PrP}^{\mathrm{d}}$ in CNS or LRS, see Table 4) but the apparent clinical progression seen in these two goats (G08-1396: development of a positive scratch test on repeated examinations; G08-1430: consistent positive scratch test and later repeated display of over-reactivity to tactile stimuli) was consistent with scrapie although the lack of other signs suggested a less advanced clinical phase than in the CNS-positive goats. Either the signs were misleading, although we were unable to establish an alternative diagnosis, or scrapie can indeed produce clinical signs suggestive of CNS involvement in the absence of detectable $\operatorname{Pr} \mathrm{P}^{\mathrm{d}}$ in the CNS by current postmortem tests.

Circling, which was seen in one goat with scrapie when blindfolded and has also been described in one case report [29], is suggestive of an asymmetrical brain lesion whereas the neuropathological changes in caprine TSEs are generally bilateral symmetrical $[10,30]$. Only half of the brain of the goat was available for histopathological examination, which prevented us from comparing the severity of lesions between both sides. Similarly, the magnitude of $\operatorname{PrP}^{\mathrm{d}}$ accumulation in the thalamus did not appear to be proportional to the frequency of pruritic behaviour or associated with a positive scratch test, even though thalamic lesions were considered to be responsible for the pruritus in caprine scrapie [10]. There was some indication that extensive $\operatorname{PrP}^{\mathrm{d}}$ accumulation in the cerebellum was associated with more severe signs of cerebellar dysfunction but one animal with a similarly high score of $\operatorname{PrP}^{\mathrm{d}}$ deposits presented without any cerebellar signs. By contrast, the severity of clinical disease, i.e. the clinical status based on the frequency and severity of expressed clinical signs, appeared to be largely proportional to the total amount of $\operatorname{PrP}^{\mathrm{d}}$ accumulation in the brain, particularly in those scrapie-affected goats that were examined and observed repeatedly prior to cull: the scrapie case without evident clinical signs of scrapie had the lowest $\operatorname{PrP}^{\mathrm{d}}$ score compared to inconclusive cases or scrapie suspects. The goat with the widest range of clinical signs (G08-1447), however, was not the one with the highest $\operatorname{PrP}^{\mathrm{d}}$ score. Similarly, the mean $\operatorname{PrP}^{\mathrm{d}}$ score was lowest in the scrapie-affected goats without evident signs of scrapie that were only assessed once by the short protocol although the range in the $\operatorname{Pr} P^{d}$ score was more variable. This variability may be due to failure to detect some scrapieassociated signs using the short protocol only (e.g. pruritus) and the fact that some animals were culled up to 57 days after the short clinical assessment, during which time $\operatorname{PrP}^{\mathrm{d}}$ accumulation in the brain may have increased.

The sometimes poor correlation between clinical disease or clinical signs in particular and detectable $\operatorname{PrP}^{\mathrm{d}}$ accumulation in the brain, which has also been documented in other species, such as mice [31], cattle [32] and pigs [33], warrants further investigations into the cause of clinical abnormalities in TSEs.

The definition of excessive pruritic behaviour expressed as pruritus score and its cut-off value of 0.5 was arbitrary and not based on any published information. In addition, we only counted each event of pruritic activity and did not consider the duration of a pruritic session, which was usually brief (approximately $85 \%$ of these lasted less than 20 seconds). This may explain why a positive scratch test was not necessarily associated with excessive pruritus. A positive correlation between pruritus (based on wool loss) and scratch test response has been reported for Irish sheep with scrapie [2], whereas this was not found in British sheep where pruritus was based on the display of pruritic activity [20]. The lack of "true" controls from a TSE-free herd - we only had exposed scrapie-negative goats - made it difficult to establish a baseline pruritic activity. In a behavioural study of sheep infested with Psoroptes ovis "increased rubbing" was defined as total time rubbing that was three standard deviations above the mean baseline rubbing time [34]. If we defined a similar cut-off point by using the data from our very limited number of scrapie-free cases with available camera recordings 
none of the goats would have been pruritic due to the extremely high pruritic score of one of the scrapie-free goats. If this animal was excluded, the resulting cut-off point of 0.32 would have made almost every scrapiepositive goat excessively pruritic. In addition, other pruritic skin conditions may have existed in these goats, although we did detect ectoparasites macroscopically only in two goats and those that presented with skin conditions (crusts on ears suggestive of mange, ringshaped hair loss suggestive of dermatomycosis but no further examination was carried out) did not display a positive scratch test. Ideally, each animal should have been kept under controlled, ectoparasite-free conditions and monitored pre- and post infection to assess changes in the pruritic activities individually but this would have only been possible in experimentally infected animals. Nevertheless, the low pruritus score in some animals (e.g. GX1030) suggests that indeed a non-pruritic form of TSEs exists in goats. The hair loss observed in many TSE-negative goats in the present study made this sign unreliable as indicator of pruritus. Unlike the Italian scrapie-affected goats that presented with thinning of hair, alopecia and skin lesions predominantly on the spinal region [35], this area was rarely affected in goats in the present study.

Signs that are reportedly frequent in goats with scrapie (in at least $80 \%$ of animals) are difficulty in milking and weight loss [35]. Yet, a poor bodily condition was significantly more frequent in scrapie-negative goats, which may be due to other diseases that were not investigated as part of this study. Difficulty in milking in those goats that were milked was also more frequent in scrapienegative goats. On the other hand, touching of the hind limbs, which may occur during milking, elicited kicking or restlessness in scrapie-affected goats, even on repeated examinations and in the vast majority of animals that were not considered to be over-reactive when the head was touched (data not shown). It was not known for both signs whether these were progressive changes, i.e. progressive loss of bodily condition and change in temperament characterised by recent difficulty in milking, because of the lack of any clinical history, which could explain why these signs did not appear to be particularly useful markers for scrapie in goats. However, comparison of the BCS between first and last clinical assessment in the 24 goats kept for a longer period did not even yield significant results between TSE-positive and negative animals (data not shown).

By contrast, weight loss was evident in four of five BSE-affected goats with a similar progression as reported for sheep with BSE [12] although the body condition score did not change. Weighing provides a more accurate estimation of loss of body mass but the problems of this technique, particularly in ruminants with the large amount of digesta in the gastrointestinal tract, has been highlighted [36].

Abnormal rising or delayed lying down was a behaviour that could have been easily missed if only clinical examinations were carried out. It is possible that conditions other than scrapie were responsible for this behaviour, for example diseases of the joints, which were not examined at necropsy. There was no clinical evidence for joint diseases although some of the goats had serum antibodies against Caprine Arthritis-Encephalitis Virus. This, however, included only one goat with abnormal rising or lying down (data not shown).

Previous experimental studies of scrapie in goats produced two different clinical phenotypes: the scratching syndrome with evident pruritic behaviour amongst other neurological signs and the nervous or drowsy syndrome where pruritus was generally absent $[9,10]$. The findings in the present study suggest that the predominant clinical sign in goats with TSE at clinical end-stage is either a positive scratch test or ataxia without signs of pruritus, which is comparable to the pruritic and ataxic type of scrapie-affected sheep reported in Japan [37]. Initial clinical signs may vary, with some animals presenting with a single sign over weeks (e.g. scrapie-affected goats G08-1373 and G08-1460), whereas others present simultaneously with a range of neurological signs (e.g. BSEaffected goat GX1035). It remains unknown what causes the difference in the clinical presentation but different scrapie strains, goat breeds and genotypes may account for the observed differences. Sheep infected with classical BSE appeared to develop a prion disease characterised by pruritus, which was regardless of prion protein genotype and route of inoculation ([12], T Konold, unpublished observation on further cases intracerebrally infected with BSE), and it was hypothesised that the clinical picture was mainly influenced by the strain. Scrapie-affected goats in herd B displayed pruritus whereas this was neither observed in any goat from herd A nor described by the farmer in previous cases on this farm, which may suggest that different strains may influence the clinical picture. Two of five goats, all of which were infected with the BSE agent, however, did not show any evidence of pruritus, and one of these displayed the drowsy form of the disease. This difference was unlikely to be caused by breed because all five goats were similar Saanen crossbreeds. It is possible that different polymorphisms of the caprine PRNP may influence the clinical presentation as it has been observed for sheep with scrapie $[2,20]$. Several $P R N P$ polymorphisms have been associated with susceptibility to infection and disease, including $\mathrm{IM}_{142}$, which produced an increased incubation period in BSE-infected goats [38]. Although this genotype also seemed to increase the incubation period in the BSE-challenged goats in our study, it had 
no effect on the clinical presentation since both the pruritic and nervous or drowsy syndrome were found in $\mathrm{IM}_{142}$ goats. Similarly, both syndromes were also found in $\mathrm{I}_{142}$ homozygous scrapie-affected goats (e.g. AngloNubian goat G08-1475: nervous syndrome; Alpine X Saanen goat G08-1147: scratching syndrome). Further studies would be required to assess whether other polymorphisms, not necessarily restricted to PRNP, may influence the clinical picture.

Drooling of saliva was observed only in one scrapieaffected goat whereas studies on a limited number of scrapie-affected goats in the UK and USA suggested that this sign was more frequent $[4,5]$. In an Italian study of 500 goats, approximately $10 \%$ of the cases displayed salivation, which was seen in the terminal phase of the disease [35]. Drooling of saliva accompanied by aspiration pneumonia may be suggestive of a dysfunction of the accessory, vagal and glossopharyngeal nerves [39]. The jaw and tongue tone appeared normal in this goat; although the goat was seen eating hay attempts to make the animal swallow by palpation of the pharynx were unsuccessful.

Our findings in the BSE-affected goats were in disagreement with earlier reports that described it a disease with short clinical duration in the absence of pruritus $[13,14]$. It is likely that the detailed clinical assessment, which included testing of the scratch response, enabled us to detect clinical cases earlier and document clinical signs that would have been missed under normal husbandry procedures. One intracerebrally infected goat presented with the drowsy form, which had previously only been reported for orally infected goats [13]. As reported for sheep [12], the clinical presentation of BSE - based on the limited number of BSE-affected animals in the present study - and scrapie appears to be largely similar in goats, with some individual variability in the clinical presentation, whereas scrapie and BSE in cattle are clinically different [40]. A deficient menace response was rare in scrapie-affected goats but it was displayed in three of five BSE-affected goats. Similarly, spontaneous head tossing during the examination was a sign predominantly seen in BSE-affected goats, possibly caused by disturbance of the trigeminal nerve sensory area as hypothesised for BSE in cattle [41]. We cannot exclude that the intracerebral inoculation of the BSE cases may have affected the pathways involving the menace response or facial sensation although this is not supported by similar studies in sheep with BSE (rarely seen regardless of route of inoculation [12]) or atypical scrapie (menace response may be absent in naturally [42] and intracerebrally infected sheep [T Konold, unpublished observation]). The topographical distribution of neuropathological changes in experimentally infected goats was found to be consistent regardless of route of inoculation [10]. The report of blindness in a goat with atypical scrapie [43] may justify the assessment of the menace response in goats.

The clinical presentation of scrapie in goats is usually described as similar to scrapie in sheep $[44,45]$ but specific differences seem to exist. Rubbing was reportedly more frequent in TSE-affected sheep than goats [35]. The present study confirmed that nibbling of body parts was the most frequent pruritic activity in goats; in a separate study in 162 scrapie-affected sheep rubbing was most frequently observed ( $\mathrm{T}$ Konold, unpublished observation). Sheep with scrapie were found to display hypoaesthesia in the limbs [3], whereas the kicking elicited by many TSE-affected goats when the hind limb were touched were suggestive of hyperaesthesia. Indeed, hyperaesthesia was considered more pronounced in goats than sheep with scrapie [46]. In addition, repeated spontaneous startle responses observed in all BSE-affected goats and some scrapie-affected goats are rarely mentioned in sheep with scrapie whereas it occurs in BSE of cattle ( $\mathrm{T}$ Konold, unpublished observation).

\section{Conclusion}

Scrapie or BSE in goats can present as clinical disease with or without pruritus; the cause for this variability remains unknown. Signs associated with scrapie (tremor, positive scratch test, extensive hair loss, ataxia and absent menace response) were highly disease-specific but if goats are scanned for scrapie based on clinical signs alone, many cases will be missed. Although the overall amount of $\operatorname{PrP}^{\mathrm{d}}$ accumulation in the brain appeared to be related to the severity of clinical disease, there was poor evidence that detectable $\operatorname{PrP}^{\mathrm{d}}$ actually causes neurological signs. Some differences in the clinical signs compared to sheep appear to exist.

\footnotetext{
Additional file 1: Short exam protocol. Short clinical examination protocol used for a brief clinical assessment of the goats as part of the herd cull.

Click here for file

[http://www.biomedcentral.com/content/supplementary/1746-6148-6-13S1.DOC ]

Additional file 2: Long exam protocol. Neurological examination protocol used for a more detailed clinical assessment of the goats. Click here for file

[ http://www.biomedcentral.com/content/supplementary/1746-6148-6-13S2.DOC]

Additional file 3: G08-1349 milking. Two goats, G08-1349 (41 monthold white Anglo-Nubian doe, scrapie-positive on brain examination) and G08-1357 (scrapie-negative, 65 month-old Toggenburg doe), are prepared for milking. Compared to the scrapie-negative doe, G08-1349 is more reluctant to enter the milk stand and lifts repeatedly its hind legs when the udder is touched or the cluster is attached. Click here for file

[ http://www.biomedcentral.com/content/supplementary/1746-6148-6-13S3.MOV]
} 
Additional file 4: G08-1407 tremor. Anglo-Nubian doe, 52 months of age, scrapie-positive on brain examination, with head tremor at rest and extensive hair loss on the poll and neck with skin lesions; also motheaten appearance of the coat (right flank).

Click here for file

[http://www.biomedcentral.com/content/supplementary/1746-6148-6-13S4.MOV]

Additional file 5: G08-1430 scratch test. Anglo-Nubian doe, 104 months old, with PrPd accumulation in LRS only. This goat raises its head and swings its head from side to side when scratched on the cranial throracic spinal region. Similar head movements with licking of the lips are elicited by merely pressing the base of the tail.

Click here for file

[http://www.biomedcentral.com/content/supplementary/1746-6148-6-13S5.MOV]

Additional file 6: G08-1389 getting up. Saanen $\times$ Anglo-Nubian doe (white, blue spray mark on the back), 51 months old, with PrP ${ }^{d}$ accumulation in LRS only displays a self-induced scratch response (head movements) when a pen mate rests and nibbles its foot on the dorsum. It is seen lying facing the camera before rising normally on its hind limbs first, followed by some pruritic behaviour (nibbling left flank):

subsequently (here: 15 days later), this goat was observed to rise abnormally on its forelimbs first. It also appears to have slight difficulty rising (rocking before getting up). Pruritic behaviour (scratching, nibbling) is again exhibited when the animal is standing. This animal never displayed a positive scratch test when scratched by the examiner Click here for file

[http://www.biomedcentral.com/content/supplementary/1746-6148-6-13S6.MOV]

Additional file 7: G08-1110 startle. This 36 month-old Anglo-Nubian doe displays frequent spontaneous startle responses when standing. Note the body flinches in the absence of any obvious external stimulus. Click here for file

[http://www.biomedcentral.com/content/supplementary/1746-6148-6-13S7.MOV]

Additional file 8: G08-1111 gait. This 36 month-old Anglo-Nubian doe is ataxic, walks with broad-based hind limbs and occasionally hops with both hind limbs. A high stepping gait is evident when the goat, which is joined by two other scrapie-affected goats (G08-1110 and G08-1112),

passes the drain cover on its way towards the camera.

Click here for file

[http://www.biomedcentral.com/content/supplementary/1746-6148-6-13S8.MOV]

Additional file 9: GX1032 menace. Saanen goat, intracerebrally inoculated with the BSE agent, at 26.5 months post inoculation. Testing of the menace response does not elicit blinking although the animal is able to blink otherwise. Note the animal's struggle when the head is

held, which is a sign of aversion to the cranial nerve testing.

Click here for file

[http://www.biomedcentral.com/content/supplementary/1746-6148-6-13S9.MOV]

Additional file 10: GX1031 scratch test. Saanen goat, intracerebrally inoculated with the BSE agent, at 34 months post inoculation. Scratching of the dorsum elicits side-to-side head movements with occasional lip licking.

Click here for file

[http://www.biomedcentral.com/content/supplementary/1746-6148-6-13S10.MOV]

Additional file 11: GX1030 lying down. Saanen goat, intracerebrally inoculated with the BSE agent, at 30.5 months post inoculation. This goat (blue spray mark " 30 ") rests on its fore limbs but hesitates lowering the hind limbs for a considerable time.

Click here for file

[http://www.biomedcentral.com/content/supplementary/1746-6148-6-13S11.MOV]

\section{Acknowledgements}

We are grateful to VLA staff of the Animal Services Unit, Pathology, Molecular Pathogenesis and Genetics and Laboratory Services who contributed to inoculation and husbandry of animals, necropsies, tissue processing and diagnostic services. We thank the TSE Programme managers, J Hope and MD Dawson, for their involvement in the herd cull and the Animal Health veterinary officers and farmers for the referral of the cases and supervision of the herd cull.

The studies would have been impossible without the financial support from Defra (TS5300, SE1956), the FSA (M03065) and the European Union (TSE Community Reference Laboratory).

\section{Author details}

${ }^{1}$ Neuropathology, Veterinary Laboratories Agency Weybridge, New Haw Addlestone, KT15 3NB, UK. ${ }^{2}$ Pathology, Veterinary Laboratories Agency Lasswade, Pentlands Science Park, Penicuik, Midlothian, EH26 OPZ, UK. ${ }^{3}$ The Roslin Institute and Royal (Dick) School of Veterinary Studies, University of Edinburgh, Roslin, Midlothian, EH25 9PS, UK. ${ }^{4}$ Molecular Pathogenesis \& Genetics 6, Veterinary Laboratories Agency Weybridge, New Haw Addlestone, KT15 3NB, UK

\section{Authors' contributions}

TK drafted the manuscript and carried out the clinical assessments. Participants in the clinical data acquisition and analysis were GEB, who also analysed the camera recordings, and LJP. LG and MMS were responsible for the pathological examinations and supported by SS who scored the brains for PrPd accumulation. The research projects were managed by MMS, LG and SACH; LJP contributed to the coordination of the herd cull. WG and SC participated in the genotyping of the goats in these projects.

All authors read and approved the final manuscript.

Received: 20 October 2009 Accepted: 4 March 2010

Published: 4 March 2010

\section{References}

1. Parry HP: Scrapie disease in sheep London: Academic PressOppenheimer DR 1983

2. Healy AM, Weavers E, McElroy M, Gomez-Parada M, Collins JD, O'Doherty E, Sweeney T, Doherty ML: The clinical neurology of scrapie in Irish sheep. J Vet Intern Med 2003, 17:908-916.

3. Vargas F, Lujan L, Bolea R, Monleon E, Martin-Burriel I, Fernandez A, De Blas I, Badiola JJ: Detection and clinical evolution of scrapie in sheep by 3rd eyelid biopsy. J Vet Intern Med 2006, 20:187-193.

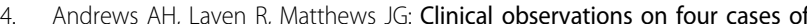
scrapie in goats. Vet Rec 1992, 130:101.

5. Hadlow WJ, Kennedy RC, Race RE, Eklund CM: Virologic and neurohistologic findings in dairy goats affected with natural scrapie. Vet Pathol 1980, 17:187-199.

6. Fankhauser R, Vandevelde M, Zwahlen R: Scrapie in der Schweiz? [Scrapie in Switzerland?]. Schweiz Arch Tierheilkd 1982, 124:227-232.

7. Harcourt RA: Naturally-occurring scrapie in goats. Vet Rec 1974, 94:504.

8. Hourrigan $J L$, Klingsporn AL, McDaniel HA, Riemenschneider MN: Natural scrapie in a goat. J Am Vet Med Assoc 1969, 154:538-539.

9. Pattison $\mathrm{IH}$, Millson GC: Scrapie produced experimentally in goats with special reference to the clinical syndrome. J Comp Pathol 1961, 71:101-109.

10. Hadlow WJ: The pathology of experimental scrapie in the dairy goat. Res Vet Sci 1961, 2:289-314.

11. Houston EF, Gravenor MB: Clinical signs in sheep experimentally infected with scrapie and BSE. Vet Rec 2003, 152:333-334.

12. Konold T, Bone G, Vidal-Diez A, Tortosa R, Davis A, Dexter G, Hill P, Jeffrey M, Simmons MM, Chaplin MJ, Bellworthy SJ, Berthelin-Baker C: Pruritus is a common feature in sheep infected with the BSE agent. BMC Vet Res 2008, 4:16.

13. Foster JD, Hope J, Fraser H: Transmission of bovine spongiform encephalopathy to sheep and goats. Vet Rec 1993, 133:339-341.

14. Foster JD, Parnham D, Chong A, Goldmann W, Hunter N: Clinical signs, histopathology and genetics of experimental transmission of BSE and natural scrapie to sheep and goats. Vet Rec 2001, 148:165-171. 
15. Eloit M, Adjou K, Coulpier M, Fontaine JJ, Hamel R, Lilin T, Messiaen S, Andréoletti $O$, Baron T, Bencsik A, Biacabé AG, Beringue $V$, Laude $H$, Le Dur A, Vilotte JL, Comoy E, Deslys JP, Grassi J, Simon S, Lantier F, Sarradin P. BSE agent signatures in a goat. Vet Rec 2005, 156:523-524.

16. Jeffrey M, Martin S, González L, Foster J, Langeveld JP, van Zijderveld FG, Grassi J, Hunter N: Immunohistochemical features of $\operatorname{PrP}(\mathrm{d})$ accumulation in natural and experimental goat transmissible spongiform encephalopathies. J Comp Pathol 2006, 134:171-181.

17. EFSA: Opinion of the Scientific Panel on Biological Hazards on a request from the European Commission on certain aspects related to the risk of Transmissible Spongiform Encephalopathies (TSEs) in ovine and caprine animals. EFSA J 2007, 466:1-10[http://www.efsa.europa.eu/en/scdocs/doc/ 466.pdf].

18. Konold T, Bone G, Simmons MM, Dexter G, Moore SJ, Pettitt RG: Scrapie in goats. Vet Rec 2007, 161:395-396.

19. Wells GAH, Hawkins SAC: Animal models of transmissible spongiform encephalopathies: Experimental infection, observation and tissue collection. Techniques in prion research Basel: Birkhäuser VerlagLehmann S, Grassi J 2004, 37-71.

20. Konold T, Bone G, Ortiz-Pelaez A, Tortosa R, Clifford D, Dexter G, Simmons MM, Spiropoulos J, Berthelin-Baker CF: Associations of clinical signs and prion protein genotypes in British sheep with scrapie. Dtsch Tierärztl Wochenschr 2009, 116:380-388.

21. Russel A: Body condition scoring of sheep. In Pract 1984, 6:91-93.

22. Dustan BH, Spencer YI, Casalone C, Brownlie J, Simmons MM: A histopathologic and immunohistochemical review of archived UK caprine scrapie cases. Vet Pathol 2008, 45:443-454.

23. Stack MJ: Western immunoblotting techniques for the study of transmissible spongiform encephalopathies. Techniques in prion research Basel: Birkhäuser VerlagLehmann S, Grassi J 2004, 97-116

24. González L, Martin S, Sisó S, Konold T, Ortiz-Peláez A, Phelan L, Goldmann W, Stewart P, Saunders G, Windl O, Jeffrey M, Hawkins SAC, Dawson $M$, Hope J: High prevalence of scrapie in a dairy goat herd: tissue distribution of disease-associated PrP and effect of PRNP genotype and age. Vet Res 2009, 40:65.

25. Mayhew IG: Neurologic evaluation. Large animal neurology Chichester, UK: Wiley-Blackwell, 2 2008, 11-46.

26. D'Angelo A, Maurella C, Bona C, Borrelli A, Caramelli M, Elena CM, Jaggy A, $\mathrm{Ru} \mathrm{G}$ : Assessment of clinical criteria to diagnose scrapie in Italy. Vet J 2007, 174:106-112.

27. Jeffrey M, Martin S, Thomson JR, Dingwall WS, Begara-McGorum I, González L: Onset and distribution of tissue PrP accumulation in scrapieaffected suffolk sheep as demonstrated by sequential necropsies and tonsillar biopsies. J Comp Pathol 2001, 125:48-57.

28. González L, Dagleish MP, Martin S, Dexter G, Steele P, Finlayson J, Jeffrey M: Diagnosis of preclinical scrapie in live sheep by the immunohistochemical examination of rectal biopsies. Vet Rec 2008, 162:397-403.

29. Laven R: Scrapie in a four year old goat. Goat Vet Soc J 1990, 11:77-79.

30. Sofianidis G, Psychas V, Billinis C, Spyrou V, Argyroudis S, Papaioannou N, Vlemmas I: Histopathological and immunohistochemical features of natural goat scrapie. J Comp Pathol 2006, 135:116-129.

31. Lasmézas Cl, Deslys JP, Robain $O$, Jaegly A, Beringue V, Peyrin JM, Fournier JG, Hauw JJ, Rossier J, Dormont D: Transmission of the BSE agent to mice in the absence of detectable abnormal prion protein. Science 1997, 275:402-405.

32. Masujin K, Matthews D, Wells GAH, Mohri S, Yokoyama T: Prions in the peripheral nerves of bovine spongiform encephalopathy-affected cattle. J Gen Virol 2007, 88:1850-1858.

33. Konold T, Spiropoulos J, Chaplin MJ, Thorne L, Spencer YI, Wells GAH, Hawkins SAC: Transmissibility studies of vacuolar changes in the rostral colliculus of pigs. BMC Vet Res 2009, 5:35.

34. Berriatua $E$, French NP, Wall R, Smith KE, Morgan KL: Within-flock transmission of sheep scab in naive sheep housed with single infested sheep. Vet Parasitol 1999, 83:277-289.

35. Capucchio MT, Guarda F, Pozzato N, Coppolino S, Caracappa S, Di Marco V: Clinical signs and diagnosis of scrapie in Italy: a comparative study in sheep and goats. J Vet Med A Physiol Pathol Clin Med 2001, 48:23-31.

36. Lawrence TLJ, Fowler VR: Measuring growth. Growth of farm animals Wallingford, UK: CABI Publishing, 2 2002, 277-319.

37. Onodera T, Saeki K: Japanese scrapie cases. Jpn J Infect Dis 2000, 53:56-61.
38. Vaccari $G$, Panagiotidis $C H$, Acin C, Peletto S, Barillet F, Acutis P, Bossers A, Langeveld J, Van Keulen L, Sklaviadis T, Badiola JJ, Andréoletti O, Groschup MH, Agrimi U, Foster J, Goldmann W: State-of-the-art review of goat TSE in the European Union, with special emphasis on PRNP genetics and epidemiology. Vet Res 2009, 40:48.

39. Mayhew IG: Dysphagia. Large animal neurology Chichester, UK: WileyBlackwell, 2 2008, 115-118.

40. Konold T, Lee YH, Stack MJ, Horrocks C, Green RB, Chaplin M, Simmons MM, Hawkins SA, Lockey R, Spiropoulos J, Wilesmith JW, Wells GA: Different prion disease phenotypes result from inoculation of cattle with two temporally separated sources of sheep scrapie from Great Britain. BMC Vet Res 2006, 2:31.

41. Austin AR, Hawkins SAC, Kelay NS, Simmons MM: New observations on the clinical signs of BSE and scrapie. Transmissible spongiform encephalopathies. Proceedings of a consultation on BSE with the Scientific Veterinary Committee of the Commission of the European Communities: 14-15 September 1993; Brussels Brussels: European Commission, AgricultureBradley R, Marchant B 1994, 277-287.

42. Konold T, Davis A, Bone G, Bracegirdle J, Everitt S, Chaplin M, Saunders GC, Cawthraw S, Simmons MM: Clinical findings in two cases of atypical scrapie in sheep: a case report. BMC Vet Res 2007, 3:2.

43. Nentwig A, Oevermann A, Heim D, Botteron C, Zellweger K, Drögemüller C, Zurbriggen A, Seuberlich T: Diversity in neuroanatomical distribution of abnormal prion protein in atypical scrapie. PLoS Pathog 2007, 3:e82.

44. Ulvund MJ: Clinical findings in scrapie. Prions in humans and animals Berlin: de GruyterHörnlimann B, Riesner D, Kretzschmar H 2006, 398-407.

45. Radostits OM, Gay CC, Hinchcliff KW, Constable PD: Diseases associated with prions. Veterinary Medicine: a textbook of the diseases of cattle, horses, sheep, pigs, and goats London, UK: Saunders-Elsevier, 10 2007, 1439-1454.

46. Wood JN, Done SH, Pritchard GC, Wooldridge MJ: Natural scrapie in goats: case histories and clinical signs. Vet Rec 1992, 131:66-68.

doi:10.1186/1746-6148-6-13

Cite this article as: Konold et al:: Monitoring of clinical signs in goats with transmissible spongiform encephalopathies. BMC Veterinary Research 2010 6:13.

\section{Submit your next manuscript to BioMed Central and take full advantage of:}

- Convenient online submission

- Thorough peer review

- No space constraints or color figure charges

- Immediate publication on acceptance

- Inclusion in PubMed, CAS, Scopus and Google Scholar

- Research which is freely available for redistribution

Submit your manuscript at www.biomedcentral com/submit
C Biomed Central 